\title{
Temporary placement of fully covered self-expandable metal stents for the treatment of benign biliary strictures
}

United European Gastroenterology Journal 2016, Vol. 4(3) 403-412 (C) Author(s) 2015 Reprints and permissions: sagepub.co.uk/journalsPermissions.nav DOI: 10.1177/2050640615606550 ueg.sagepub.com

(S)AGE

\author{
Ulriikka Chaput ${ }^{1}$, Ariane Vienne ${ }^{1}$, Etienne Audureau ${ }^{2}$, Paul Bauret ${ }^{3}$, \\ Philippe Bichard ${ }^{4}$, Dimitri Coumaros ${ }^{5}$, Bertrand Napoléon ${ }^{6}$, Thierry Ponchon ${ }^{7}$, \\ Jean-Christophe Duchmann ${ }^{8}$, René Laugier ${ }^{9}$, Hervé Lamouliatte ${ }^{10}$, \\ Bruno Védrenne ${ }^{11}$, Marianne Gaudric ${ }^{1}$, Stanislas Chaussade ${ }^{1,12}$, \\ Françoise Robin ${ }^{13}$, Sarah Leblanc ${ }^{1}$ and Frédéric Prat ${ }^{1,12}$
}

\begin{abstract}
Background: Endoscopic treatment of benign biliary strictures (BBS) can be challenging.

Objective: To evaluate the efficacy of fully covered self-expandable metal stents (FCSEMS) in BBS.

Methods: Ninety-two consecutive patients with BBS (chronic pancreatitis $(n=42)$, anastomotic after liver transplantation $(n=36)$, and post biliary surgical procedure $(n=14))$ were included. FCSEMS were placed across strictures for 6 months before endoscopic extraction. Early success rate was defined as the absence of biliary stricture or as a minimal residual anomaly on post-stent removal endoscopic retrograde cholangiopancreatography (ERCP). Secondary outcomes were the final success and stricture recurrence rates as well as procedure-related morbidity.

Results: Stenting was successful in all patients. Stenting associated complications were minor and occurred in $22(23.9 \%)$ patients. Migration occurred in $23(25 \%)$ patients. Stent extraction was successful in all but two patients with proximal stent migration. ERCP after the 6 months stenting showed an early success in $84.9 \%$ patients (chronic pancreatitis patients: $94.7 \%$, liver transplant: $87.9 \%$, post-surgical: $61.5 \%)(p=0.01)$. Final success was observed in $57 / 73(78.1 \%)$ patients with a median follow-up of $12 \pm 3.56$ months. Recurrence of biliary stricture occurred in 16/73 (21.9\%) patients.

Conclusions: FCSEMS placement is efficient for patients with BBS, in particular for chronic pancreatitis patients. Stent extraction after 6 months indwelling, although generally feasible, may fail in a few cases.
\end{abstract}

Keywords

Benign biliary strictures, metal stents, chronic pancreatitis, liver transplantation, endoscopic retrograde cholangiopancreatography, FCSEMS

Received: 26 March 2015; accepted: 23 August 2015

\section{Introduction}

Benign biliary strictures (BBS) may result from various causes, the most common being intra-operative biliary injury during cholecystectomy, inflammation and fibrosis in the course of chronic pancreatitis, and anastomotic strictures mainly due to ischemia and graft conservation impairment after liver transplantation. In the past decades, endoscopic retrograde cholangiopancreatography (ERCP) and stenting have been preferred as the first line treatment for BBS in consideration of their high initial success rate and morbidity

\footnotetext{
${ }^{1}$ Gastroenterology Department, Cochin Hospital, Paris, France

${ }^{2}$ Public Health Department, Henri Mondor Hospital, LIC EA4393 Paris Est Créteil University (UPEC), Paris, France

${ }^{3}$ Gastroenterology Department, Saint Eloi Hospital, Montpellier, France

${ }^{4}$ Gastroenterology Department, La Tronche Hospital, Grenoble, France

${ }^{5}$ Gastroenterology Department, Civil Hospital of Strasbourg, Strasbourg, France

${ }^{6}$ Gastroenterology Department, Private Hospital Jean Mermoz, Lyon, France ${ }^{7}$ Gastroenterology Department, Edouard Herriot Hospital, Lyon, France

${ }^{8}$ Gastroenterology Department, Hospital of Compiègne, Compiègne, France ${ }^{9}$ Gastroenterology Department, La Timone Hospital, Marseille, France

${ }^{10}$ Gastroenterology Department, Saint-André Hospital, Bordeaux, France

${ }^{11}$ Gastroenterology Department, Emile Muller Hospital, Mulhouse, France

${ }^{12}$ Paris-Descartes University, Paris, France

${ }^{13}$ French Society of Digestive Endoscopy, Lyon and Paris, France

Corresponding author:

Frédéric Prat, Gastroenterology Department, Cochin Hospital, Paris, France. Email: frederic.prat@cch.aphp.fr
} 
lower than surgery. Until recently, the standard of care for the endoscopic management of BBS was using one or several plastic stents exchanged serially over a period of approximately one year. ${ }^{1-3}$ Circa 2008, the advent of removable fully covered self-expandable metal stents (FCSEMS) opened a new paradigm for BBS management. ${ }^{4}$ After initial studies demonstrated the feasibility of short temporary stenting with partially covered selfexpandable stents, ${ }^{5}$ and encouraging results were found in specific conditions such as post-liver transplant strictures, ${ }^{6,7}$ outcomes of a modified strategy consisting in using only one, large bore FCSEMS for a shorter period of time with no intermediate exchange needed to be assessed in patients responding to the broader definition of common bile duct BBS. The present study was designed to evaluate prospectively the outcomes of the temporary placement of FCSEMS for benign biliary strictures in a multicenter setting.

\section{Materials and methods}

\section{Patients}

This study was conducted prospectively in ten French hospitals, under the aegis of the French Society of Digestive Endoscopy (SFED). Consecutive patients presenting with a BBS were considered for temporary placement of FCSEMS and included after informed consent was obtained. Inclusion criteria were a history of liver transplantation, chronic pancreatitis or biliary surgery, sustained impairment of liver function tests and a biliary stricture associated with ductal dilation detected by ultrasonography (US), computed tomography (CT), or magnetic resonance imaging (MRI), with a minimum distance of $2 \mathrm{~cm}$ between the upper stricture's edge and the lower limit of the main biliary confluence or liver hilum. Exclusion criteria were an uncertainty about the benign nature of the stricture, an intra-hepatic cholangitis and/or a stricture extending beyond the hilum, and previous interventions using metal stents. The study received IRB approval by the Comité de Protection des Personnes Ile de France 3 and received clearance by the AFSSAPS (2006-A00197-44).

\section{Endoscopic protocol}

Briefly, all procedures were performed with a largechannel duodenoscope under deep sedation using propofol. After selective biliary cannulation, a cholangiogram was obtained to evaluate the location and the severity of the stricture. Permalume ${ }^{\circledR}$-fully covered Wallflex ${ }^{\circledR}$ stents (Boston Scientific, $10 \mathrm{~mm}$ in diameter, 40,60 , or $80 \mathrm{~mm}$ in length) were used in all patients. The choice of stent length was adjusted to the anatomy of the common bile duct and the length and level of the stricture. The proximal stent end had to be placed below the main hepatic confluence, in order to prevent occlusion of one biliary branch by the stent cover. Performing a biliary sphincterotomy was not mandatory, but was usually done to allow for a full expansion of the stent through the papilla, make extraction easier and prevent pancreatitis due to pancreatic duct occlusion by the stent cover. Stricture dilation with a balloon before placing the stent was not recommended systematically but was allowed, especially in case of a tight stricture with difficult passage of a catheter.

FCSEMS were left in place for 6 months and then removed using a snare and/or a rat tooth forceps, by grasping the metallic loop at the distal end of the stent. As to assess biliary patency, a cholangiogram was performed immediately after stent removal, by using a $12 \mathrm{~mm}$ retrieval balloon passed downwards through the stricture with contrast liquid being injected above the balloon. The bile duct was deemed patent with satisfactory sizing when the fully inflated balloon was passed without any significant resistance and if there was no evidence of contrast retention above the initial stricture site. Antibiotics were systematically administered prophylactically before each ERCP.

\section{Follow-up}

Patients were followed through a combination of clinical examination and liver function tests at 1, 3, 6, 9, and 12 months after FCSEMS removal. In case of recurrent stricture, follow-up was stopped and the patient was offered a new treatment whose choice was left to the investigator's decision.

\section{Statistical analysis}

Descriptive results are reported as means ( \pm standard deviation (SD)) or medians (range and inter-quartile range) for continuous variables, and as count (percentage) for categorical data. Univariate analysis of the patient or procedures' characteristics associated with outcomes of interest (i.e. initial success, migration, recurrence) were performed using $\mathrm{Chi}^{2}$ tests or Fisher's exact test for categorical variables, and unpaired t-tests or Wilcoxon rank-sum tests for continuous variables to account for non-normality of the variable distributions when demonstrated by the use of the Shapiro-Wilk test for normality. Multivariate analysis was conducted for potential predictors associated at the $p<0.2$ level in univariate analysis using Firth's bias correction to account for the small sample size. A stepwise approach was followed by removing nonsignificant variables at each step. Censored data (time to recurrence as the endpoint) were analyzed using univariate survival analysis by the log-rank test and 
multivariate Cox proportional hazards models fitted to assess the independent role of each predictor. A $p$ value $<0.05$ was considered statistically significant. All statistical analyses were performed using Stata version 12.1 (StataCorp, College Station, TX, USA). Based on previously reported results and our own experience, a sample size of at least 80 subjects was required to estimate the primary endpoint with an accuracy of $\pm 10 \%$, assuming an expected early success rate of at least $70 \%$.

\section{Results}

Ninety-two patients (68 men, 24 women) aged $54.4 \pm 9.9$ years (median 55; range 15-78) were included in ten centers (a mean of $9.2 \pm 12$ patients per center). The etiology of the biliary stricture was chronic pancreatitis $(n=42)$, anastomotic after liver transplantation $(n=36)$ and post-surgical biliary procedure, mainly cholecystectomy $(n=14)$. Forty-five $(48.9 \%)$ patients had at least one previous endoscopic treatment of the same stricture with dilation and/or plastic stenting. Table 1 summarizes endoscopic treatments prior to inclusion in the study.

FCSEMS placement was performed successfully in all patients. Twenty-six $(28.3 \%)$ patients underwent an endoscopic balloon dilation before stenting, at a median diameter of $8 \mathrm{~mm} \quad($ mean $=7.44 \pm 0.92 \mathrm{~mm}$, range 6-8).

Icterus resolved in all jaundiced patients after stenting, and liver function tests improved in all other patients. Complications occurring within five days of stenting were observed in $11(11.9 \%)$ patients, all of which were minor. Five patients, all of whom had undergone a standard $(n=3)$ or precut $(n=2)$ sphincterotomy developed a minor post-ERCP pancreatitis. One patient presented a minor hemorrhage treated endoscopically per procedure by a local injection of epinephrine. Four patients had transient abdominal pain without hyperlipasemia and one developed a cholecystitis which promptly responded to antibiotics and did not require surgery.

Table 1. Endoscopic treatments prior to inclusion in the study

\begin{tabular}{lc}
\hline No previous treatment & $47(51.1 \%)$ \\
Previous treatment & $45(48.9 \%)$ \\
Plastic stent alone & $33(35.8 \%)$ \\
Balloon dilation alone & $2(2.1 \%)$ \\
Balloon dilation associated & $16(17.4 \%)$ \\
$\quad$ with plastic stent & \\
Mean number of previous & $0.8( \pm 1.13) ; 0(0-6)$ \\
ERCP (SD); median (range) & \\
\hline
\end{tabular}

Delayed complications (between five days and 6 months) occurred in $11(11.9 \%)$ patients. Hepatobiliary infection occurred in the form of cholangitis in six patients and liver abscess in one. Biological abnormalities (cholestasis and/or jaundice) without any clinical sign occurred in four other patients.

Stent migration occurred in $23(25 \%)$ patients. The stent migrated distally in eighteen patients: either completely (the stent was no more attached to the papilla) in twelve patients, or partially (stent dislodged below the stricture but still attached to the papilla) in six patients. Proximal migration (stent dislodged above the stricture) occurred in five cases. Twelve out of 23 patients $(52.2 \%)$ with stent migration presented a persistent stricture requiring further endoscopic treatment. Most persistent strictures were post orthotopic liver transplantation (OLT) $(n=5)$ or post cholecystectomy $(n=6)$, only one stricture was due to chronic pancreatitis. These clinically significant stent migrations were observed a mean of $20.8 \pm 8$ weeks after stent implantation. Nine of $23(39.1 \%)$ patients with stent migration had undergone balloon dilation at the time of stenting.

Stent removal was possible in all but two patients with proximal migration of the stent. One of them presented with a severe chronic pancreatitis and developed a duodenal obstruction secondary to cystic dystrophy, in which the stent is unlikely to have any causal relationship. Duodenal obstruction made it impossible to remove the stent endoscopically, and the patient subsequently underwent a Whipple's procedure, during which the stent was removed. The other one died later from cardiovascular disease, with no biliary symptom or complication. Removal was found to be demanding in two types of situation: impaction of the distal end of the stent in the papilla $(n=2)$ and proximal migration $(n=2)$ of the stent. Argon Plasma destruction of the hyperplastic tissue covering the distal end of the stent was required in two patients to successfully achieve stent extraction.

Early success of endoscopic treatment, was $84.9 \%$ per-protocol $(n=73)$, but $79.3 \%$ in an intention-totreat (ITT) perspective. Success rates relative to the etiology of BBS were $87.9 \%, 94.7 \%$, and $61.5 \%$ in patients with BBS due to liver transplantation, chronic pancreatitis, and post-surgical, respectively. ERCP after the 6 months sizing period showed a persistent stricture in $11(11.9 \%)$ patients, ten of whom had had a partial $(n=3)$ or a complete $(n=7)$ distal migration of the metal stent. Table 2 displays outcomes after the stenting period.

Final success defined as a sustained biliary stricture resolution was observed in $57 / 73(78.1 \%)$ patients (61.9\% from an ITT perspective) with a median follow-up of $12( \pm 3.56 \mathrm{SD})$ months. Recurrence of biliary stricture after early success occurred in 16/73 
$(21.9 \%)$ patients. All recurrences developed at the original site of the stenosis. In particular, there was no evidence of a new stenosis at the proximal edge of the original FCSEMS. The mean time to recurrence of biliary stricture was $4.2( \pm 3.3 \mathrm{SD}$, range $0.5-12)$ months. Table 3 displays the outcomes at the end of follow-up. One patient died of pulmonary embolism before any treatment; data from two cases were unavailable. Overall outcomes are summarized in Figure 1.

Univariate statistical analysis showed that stent migration was associated with female sex $(p=0.013)$, post-transplant and other post-surgical BBS ( $p=$ $0.006)$, and a higher number of endoscopic procedures before FCSEMS placement $(p=0.026)$, such as endoscopic dilations $(p=0.025)$. However, endoscopic dilation at the time of FCSEMS placement was not associated with more stent migrations (see

Table 2. Outcomes and management at the end of the 6 months stenting period

\begin{tabular}{|c|c|c|}
\hline ERCP findings & $n(\%)$ patients & Management \\
\hline $\begin{array}{l}\text { Failed stent } \\
\text { extraction }\end{array}$ & $2(2.3 \%)$ & $\begin{array}{l}\text { Surgery, } n=1 \\
\quad \text { conservative, } n=1\end{array}$ \\
\hline Persistent & $11(13.25 \%)$ & Dilation, $n=1$ \\
\hline stricture & & $\begin{array}{l}\text { Dilation + plastic } \\
\text { stent, } n=1 \\
\text { Dilation }+2 \text { nd FCSEMS, } n=3 \\
\text { Plastic stent, } n=1 \\
\quad \text { 2nd FCSEMS, } n=5\end{array}$ \\
\hline $\begin{array}{c}\text { Minimal residual } \\
\text { anomaly }\end{array}$ & $46(53.5 \%)$ & Observation \\
\hline No stricture & $27(31.4 \%)$ & Observation \\
\hline Early success & 73 (84.9\%) & Observation \\
\hline
\end{tabular}

Supplementary table 1 online). In the multivariate analysis of risk factors for stent migration, the etiology of the stricture was independently predictive, chronic pancreatitis being a protective factor (Table 4).

Risk factors for the persistence of a stricture in the univariate analysis were found to be female sex $(p=0.04)$, stent migration $\left(p<10^{-4}\right)$, and the etiology of the stricture $(p=0.01)$. A significantly better stricture resolution rate was found in patients with chronic pancreatitis $(94.7 \%)$ than in those with liver transplant $(87.9 \%)$ and post-surgical $(61.5 \%)(p=0.01)$ strictures. However, the difference between chronic pancreatitis patients and transplanted patients did not reach significance $(p=0.33$, odds ratio $(\mathrm{OR})=2.23$, confidence interval (CI) 0.44-11.3) probably due to the small sample size (See Supplementary table 2 online). In a multivariate analysis, etiology was the only significant variable associated with early success with better results for chronic pancreatitis patients compared to postsurgical patients and liver transplant patients $(p=$ 0.021, OR $=15.5$, CI 1.5-160.4) (Table 5).

Finally, stricture recurrence was associated in univariate analysis with dilation before stent placement ( $p=0.009$, hazard ratio (HR) 3.68, CI 1.37-9.91) and stricture etiology. Higher recurrence rates were found in post-surgical ( $p=0.007$, HR 7.87 , CI $1.76-35.28)$ and post-transplant strictures $(p=0.04$, HR 3.99 , CI 1.08-14.73) when compared to chronic pancreatitis strictures. No variable was found to be independently associated with stricture recurrence in the multivariate analysis (Table 6).

\section{Discussion}

ERCP has recently become the first line treatment of benign biliary strictures as a reward of a better safety

Table 3. Outcomes at the end of the follow-up (1 year after stent extraction)

\begin{tabular}{lllr}
\hline ERCP findings & $n(\%)$ & Management & $n$ \\
\hline Stricture recurrence & $16 / 73(21.9 \%)$ & Dilation + plastic stent & 3 \\
& & 2nd FCSEMS alone & 3 \\
& & Dilation + 2nd FCSEMS & 4 \\
& & Surgical treatment & 2 \\
& & Unavailable data & 1 \\
Final success (sustained & Death from other cause & \\
$\quad$ stricture resolution): & & Observation & \\
- Per-protocol & & & \\
- ITT & $57 / 73(78.1 \%)$ & & \\
\hline
\end{tabular}




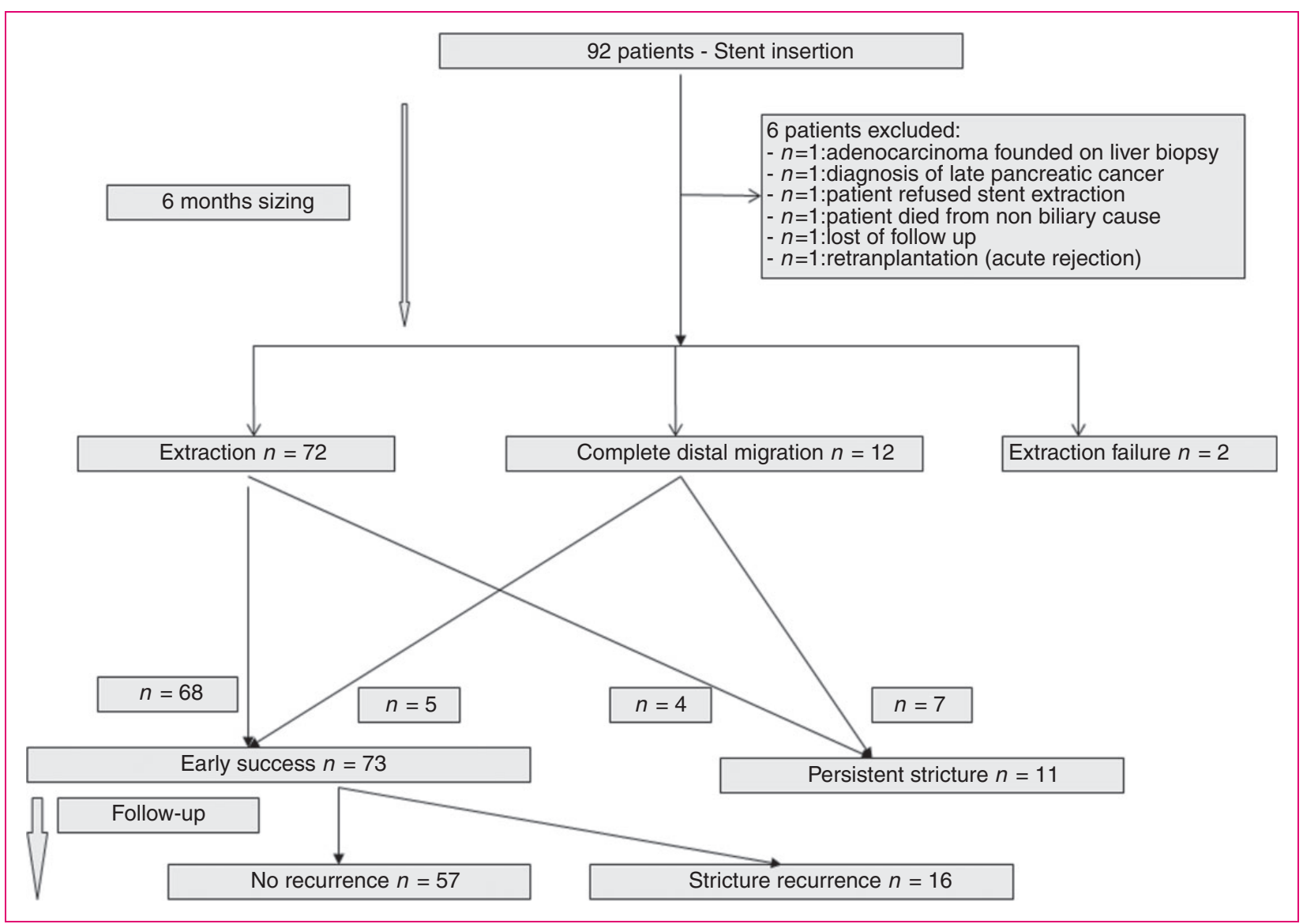

Figure 1. Flow chart summarizing overall outcomes.

Table 4. Multivariate analysis of patient's and procedural's characteristics associated with stent migration

\begin{tabular}{|c|c|c|c|}
\hline & & $p$-value & $95 \% \mathrm{Cl}$ \\
\hline Age & & NS & \\
\hline \multirow[t]{2}{*}{ Sex } & Male & NS & \\
\hline & Female & & \\
\hline \multirow[t]{3}{*}{ Etiology } & Liver transplantation & 0.009 & $1.5-16.34$ \\
\hline & Chronic pancreatitis & 1 reference & \\
\hline & Post-surgical & 0.009 & $1.62-28.77$ \\
\hline No. of previous endoscopic treatments & & NS & \\
\hline \multirow[t]{2}{*}{ Dilation in a previous procedure } & No & NS & \\
\hline & Yes & & \\
\hline \multirow[t]{2}{*}{ Dilatation preceding FCSEMS placement } & No & NS & \\
\hline & Yes & & \\
\hline \multirow[t]{2}{*}{ Dilation diameter } & $6 \mathrm{~mm}$ & NS & \\
\hline & $8 \mathrm{~mm}$ & & \\
\hline Stricture length & & NS & \\
\hline \multirow[t]{3}{*}{ FCSEMS length } & $40 \times 10 \mathrm{~mm}$ & NS & \\
\hline & $60 \times 10 \mathrm{~mm}$ & & \\
\hline & $80 \times 10 \mathrm{~mm}$ & & \\
\hline
\end{tabular}


Table 5. Multivariate analysis of patients' or procedure's characteristics associated with early success

\begin{tabular}{llll}
\hline & & N-value & $95 \% \mathrm{Cl}$ \\
\hline Age & & NS \\
Sex & Male & NS \\
& Female & \\
Etiology & Liver transplantation & 0.835 & \\
& Chronic pancreatitis & 1 reference & \\
& Post-surgical & $\mathbf{0 . 0 2 1}$ & $\mathbf{1 . 5}-160.45$ \\
Number of previous endoscopic treatments & & NS \\
Dilation in a previous procedure & No & NS \\
& Yes & \\
Dilatation preceding FCSEMS placement & No & NS \\
Dilation diameter & Yes & \\
(mm) & 6 & NS \\
Stricture length & 8 & \\
FCSEMS length & & NS \\
& $40 \times 10$ & NS \\
Migration & $60 \times 10$ & \\
& $80 \times 10$ & NS \\
& No & \\
& Yes & \\
& &
\end{tabular}

Table 6. Univariate analysis of patient's or procedure's characteristics associated with final success

\begin{tabular}{|c|c|c|c|c|c|c|}
\hline \multirow{2}{*}{$\begin{array}{l}\text { Variable } \\
\text { Age }\end{array}$} & & \multirow{2}{*}{$\begin{array}{l}n \\
69\end{array}$} & \multirow{2}{*}{$\begin{array}{l}p \text {-value } \\
0.753\end{array}$} & \multicolumn{3}{|c|}{ Hazard ratio IC $95 \%$} \\
\hline & & & & 0.992 & 0.943 & 1.043 \\
\hline \multirow[t]{2}{*}{ Sex } & Male & 51 & & 1 reference & & \\
\hline & Female & 18 & 0.5735 & 1.35 & 0.47 & 3.9 \\
\hline \multirow[t]{3}{*}{ Etiology } & Liver transplantation & 28 & 0.0381 & 3.99 & 1.08 & 14.73 \\
\hline & Chronic pancreatitis & 33 & & 1 reference & & \\
\hline & Post-surgical & 8 & 0.007 & 7.87 & 1.76 & 35.28 \\
\hline Number of previous endoscopic treatments & & 69 & 0.1451 & 1.425 & 0.885 & 2.295 \\
\hline \multirow[t]{2}{*}{ Dilation in a previous procedure } & No & 50 & & 1 reference & & \\
\hline & Yes & 11 & 0.8622 & 1.12 & 0.32 & 3.96 \\
\hline \multirow[t]{2}{*}{ Dilation preceding FCSEMS } & No & 45 & & 1 reference & & \\
\hline & Yes & 20 & 0.0098 & 3.68 & 1.37 & 9.91 \\
\hline \multirow[t]{2}{*}{ Dilation diameter (mm) } & 6 & 5 & & 1 reference & & \\
\hline & 8 & 10 & 0.1778 & 0.38 & 0.09 & 1.55 \\
\hline Stricture length & & 61 & 0.1521 & 0.614 & 0.315 & 1.197 \\
\hline \multirow[t]{3}{*}{ Stent length } & $40 \times 10$ & 19 & & 1 reference & & \\
\hline & $60 \times 10$ & 17 & 0.1718 & 4.61 & 0.52 & 41.24 \\
\hline & $80 \times 10$ & 28 & 0.0385 & 8.69 & 1.12 & 67.37 \\
\hline \multirow[t]{2}{*}{ Migration } & No & 59 & & 1 reference & & \\
\hline & Yes & 10 & 0.5945 & 1.41 & 0.4 & 4.94 \\
\hline \multirow[t]{2}{*}{ Persistent stricture } & Attenuated & 43 & 0.8855 & 1.08 & 0.39 & 2.97 \\
\hline & No & 26 & & 1 reference & & \\
\hline
\end{tabular}


profile than interventional radiology and surgery, but its best modalities are still debated. The simultaneous and temporary placement of multiple plastic stents is the current standard, as recently recommended by ESGE. This modality showed good long term results, usually over $70 \%$, except in studies including patients with chronic pancreatitis which reported long term success of $31-62 \%{ }^{8,9}$ However, this approach, requiring frequent endoscopic procedures, usually every 3 months during one year, can be impaired by poor compliance and intercurrent side effects, thus increasing morbidity and raising costs. Fully covered metal stents were developed in the 2000s initially for the prevention of tumor growth during palliative stenting. Unlike uncovered stents used for malignant conditions, FCSEMS can be extracted and therefore placed for a limited period of time in benign strictures. Recent data from prospective studies have been encouraging, with stricture resolution rates at stent removal of 43$90 \%$, although recurrences occurred in 6-47\%., $4,7,10-15$ A summary of major results from those studies is displayed in Table 7.

Our study included a large number of patients from both academic and community hospitals as well as private clinics, thus representative of the full range of BBS patients, all treated along a standardized endoscopic protocol and using only one type of FCSEMS. Some predictable drop-outs were due to initially unsuspected diagnoses of malignancy and the unexpected need for surgery during follow-up, but only one patient was lost at follow-up during the stenting period while less than $10 \%$ were lost during follow-up after stent removal. The main study limitation was the non-randomized design, making comparisons with conventional treatment outcomes (i.e. plastic stenting) speculative.

In this study, the mean time to recurrence of biliary stricture was relatively short $(4.2 \pm 3.3 \mathrm{SD}$, range $0.5-$ 12 months). However, the early success (85\%) and long term success $(78.1 \%)$ rates observed in our study are similar to those previously reported with FCSEMS and compare favorably with those of plastic stenting. ${ }^{16}$ Moreover, most recurrences occur within the first year of plastic stent removal, which is consistent with our findings. The main hindrance to the efficacy of FCSEMS in BBS appeared to be stent migration, observed in nearly $25 \%$ of patients, with a particular burden from distal migrations. It is noteworthy that nearly all (10 out of 11) strictures persistent at the end of the stenting were in patients with stent migration, although only $55 \%$ of migrations led to a persistent stricture, thus suggesting that the stent can remodel the bile duct in certain cases even when it does not stay in place as long as expected. Migration was more frequent in patients with short strictures due to liver transplantation or after cholecystectomy than in patients with BBS during chronic pancreatitis, thus possibly explaining in part the better results in chronic pancreatitis patients. In liver transplant and also some postsurgery BBS, migration is probably more likely when the stricture is at the same time short and close to the liver hilum, leading to an imbalance in the length of stent above and below the stricture. Multiple plastic stents or FCSEMS equipped with specific antimigration features could be preferred for such cases, were this observation be confirmed by further studies. Migration could also be favored by dilation at the time of stenting, but our findings do not support this hypothesis, since only dilations performed during previous endoscopic therapies were associated with a higher migration rate, whereas only $39 \%$ of patients with stent migration had undergone balloon dilation at the time of stenting. Whether or not to dilate the stricture before implanting an FCSEMS remains therefore open to the endoscopist's choice, with dilation to be considered when the stricture is very tight or its location too close to the hilum to allow for expansion and self-shortening without stent dislocation. Park et al. reported a series of 22 patients treated with metal stents with anti-migration flaps. ${ }^{11}$ No case of migration was observed in this series, but the rate of post-stenting pancreatitis was unusually high at $18 \%$. Flared-end stents did not appear to reduce the migration rate up to $30-40 \%$ in two studies. ${ }^{11,12}$ Of several other solutions proposed in order to reduce the migration rate of FCSEMS (placing the stent without prior sphincterotomy, using systematically long FCSEMS or stitching the stent to the duodenal mucosa by using clips), none has been validated by prospective studies.

It is noteworthy that nearly $50 \%$ of patients in this study had a refractory stricture, defined as a stricture which had not responded to one or several previous endoscopic treatment. This study shows that metal stents can be used as a second line treatment and still present a good efficacy profile. An important finding is that chronic pancreatitis patients $(45.6 \%$ of the patients included), whose strictures are deemed difficult to treat with plastic stents, had favorable outcomes and may therefore benefit from this type of stent, not least because they might be easier to convince of this expeditious, two-step treatment. On the contrary, it was bewildering to find transplantation and biliary surgery-associated strictures bearing a significantly poorer prognosis in terms of long term success rate than chronic pancreatitis strictures, although secondline stentings were not significantly less common in the latter subgroup ( $43 \%$ vs $48 \%$ ). However, despite several reports of lower stricture resolution rates among chronic pancreatitis patients, ${ }^{4,12}$ one recent international study found a $79.7 \%$ stricture resolution rate after 10-12 months of FCSEMS stenting in this 


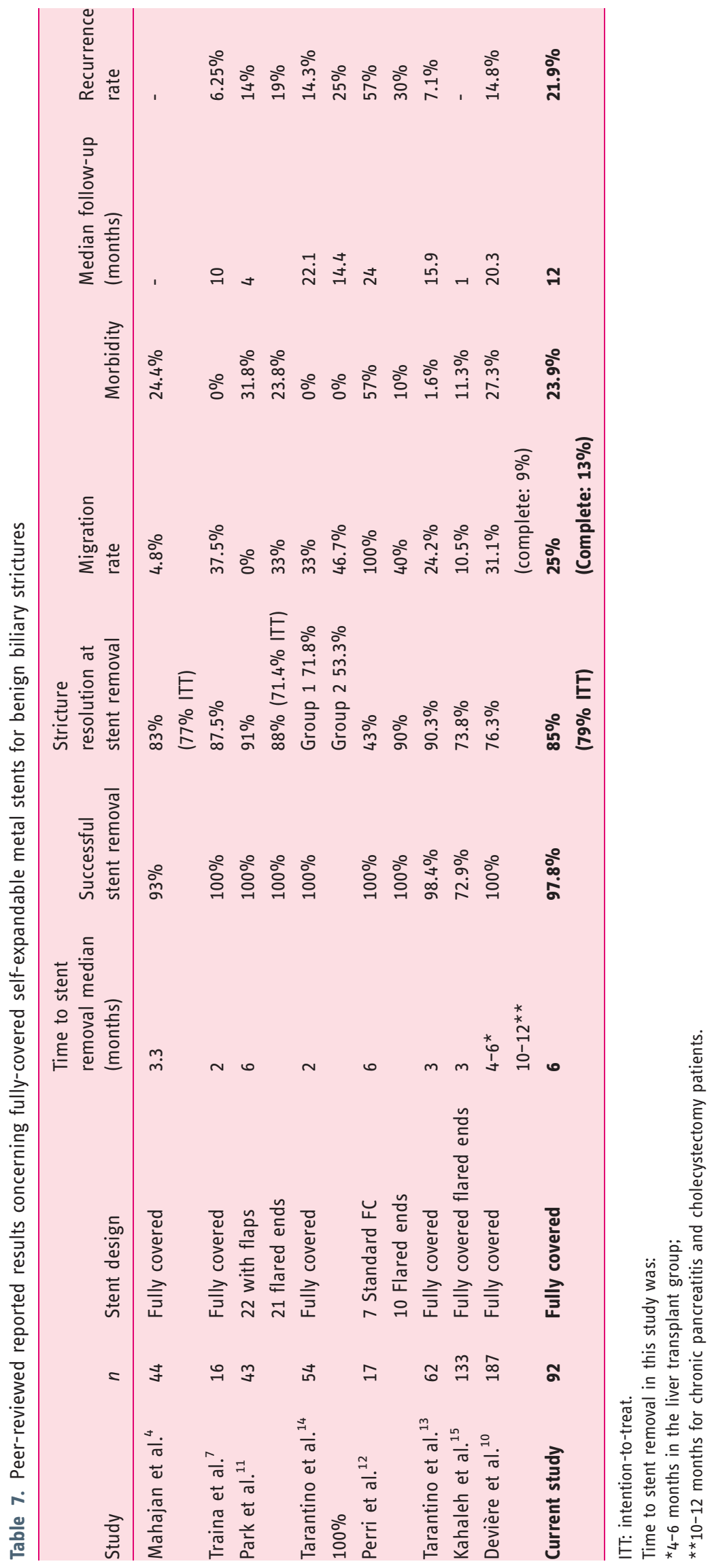


subset of patients, ${ }^{10}$ who could thus benefit from an endoscopic treatment with a metal stent and only two procedures. The main reason for poorer outcomes in OLT patients, in contrast to other studies in which somehow better results were reported with FCSEMS, ${ }^{7,13}$ can be related to the particular nature of OLT strictures and also to a higher stent migration rate in this subgroup. A different composition of anastomotic strictures in fibroblasts, inflammatory cells and elastic fibers may account for a higher elasticity and resilience of those strictures, when compared to postcholecystectomy and chronic pancreatitis ones. However, pathologic studies are lacking to support this hypothesis. Another hypothesis may result from the observation that most anastomoses were performed pretty close to the hilum, with only a short portion of the graft's common bile duct available for stent implantation above the stricture and below the main confluence, an anatomic feature which makes stents prone to migration.

Finally, stenting duration might crucially influence outcomes. Stent migration was found to negatively affect outcome primarily by reducing the duration of stenting. One could also think that a protracted stenting period would yield better results, as suggested by the experience of plastic stents, yielding better outcomes after 1 year than after shorter periods. We chose a period of 6 months because we had enough experience with the stents used in the study to have little doubt as to their removability after this timespan, whereas 2 or 3 months was more common in previous studies. ${ }^{4,6,7,13,14} \mathrm{We}$ were also aware that a serious matter for concern is removability of stents after a long indwelling, if the stent cover is too damaged to prevent impaction in hyperplasia and fibrotic-inflammatory tissue, a problem commonly encountered with partially covered stents. In the above quoted study by Devière et al., ${ }^{10}$ after a stenting period of $10-12$ months, $4 \%$ of scheduled stent removals needed multiple attempts due to difficult extraction and $14 \%$ required an anticipated removal due to complications. In that study the stricture resolution rate after stent removal was $76.3 \%$, as against $79.3 \%$ in the present study, suggesting that a longer stenting is not necessarily associated with better outcomes.

In conclusion, FCSEMS placement is feasible and efficient for patients with BBS in nearly 4 out of 5 patients, including those with previous standard endoscopic treatments. FCSEMS removal after 6 months of implantation is generally easy, but there is a caveat on the uncommon event of a failure to remove an impacted stent. Best results seem to be obtained in chronic pancreatitis patients, whereas outcomes in other types of strictures (post-OLT and cholecystectomy) may be hampered by more frequent stent migration. Better initial and long term results could be expected if stent migration rates were lower, a goal that improving stents' design is more likely to achieve than modifying endoscopic protocols.

\section{Funding}

This research received no specific grant from any funding agency in the public, commercial, or not-for-profit sectors.

\section{Conflict of interest}

None declared

\section{References}

1. Costamagna G, Pandolfi M, Mutignani M, et al. Longterm results of endoscopic management of postoperative bile duct strictures with increasing numbers of stents. Gastrointest Endosc 2001; 54: 162-168.

2. Pasha SF, Harrison ME, Das A, et al. Endoscopic treatment of anastomotic biliary strictures after deceased donor liver transplantation: outcomes after maximal stent therapy. Gastrointest Endosc 2007; 66: 44-51.

3. Poley JW, Lekkerkerker MN, Metselaar HJ, et al. Clinical outcome of progressive stenting in patients with anastomotic strictures after orthotopic liver transplantation. Endoscopy 2013; 45: 567-570.

4. Mahajan A, Ho H, Sauer B, et al. Temporary placement of fully covered self-expandable metal stents in benign biliary strictures: midterm evaluation (with video). Gastrointest Endosc 2009; 70: 303-309.

5. Kahaleh M, Tokar J, Le T, et al. Removal of self-expandable metallic Wallstents. Gastrointest Endosc 2004; 60: 640-644.

6. Chaput U, Scatton O, Bichard P, et al. Temporary placement of partially covered self-expandable metal stents for anastomotic biliary strictures after liver transplantation: a prospective, multicenter study. Gastrointest Endosc 2010; 72: 1167-1174.

7. Traina M, Tarantino I, Barresi L, et al. Efficacy and safety of fully covered self-expandable metallic stents in biliary complications after liver transplantation: a preliminary study. Liver Transpl 2009; 15: 1493-1498.

8. Catalano MF, Linder JD, George S, et al. Treatment of symptomatic distal common bile duct stenosis secondary to chronic pancreatitis: comparison of single vs. multiple simultaneous stents. Gastrointest Endosc 2004; 60: 945-952.

9. Eickhoff A, Jakobs R, Leonhardt A, et al. Endoscopic stenting for common bile duct stenoses in chronic pancreatitis: results and impact on long-term outcome. Eur J Gastroenterol Hepatol 2001; 13: 1161-1167.

10. Devière J, Reddy DN, Puspok A, et al. Successful management of benign biliary strictures with fully covered self-expanding metal stents. Gastroenterology 2014; 147 : 385-395.

11. Park do H, Lee SS, Lee TH, et al. Anchoring flap versus flared end, fully covered self-expandable metal stents to prevent migration in patients with benign biliary 
strictures: a multicenter, prospective, comparative pilot study (with videos). Gastrointest Endosc 2011; 73: 64-70.

12. Perri V, Boskoski I, Tringali A, et al. Fully covered selfexpandable metal stents in biliary strictures caused by chronic pancreatitis not responding to plastic stenting: a prospective study with 2 years of follow-up. Gastrointest Endosc 2012; 75: 1271-1277.

13. Tarantino I, Mangiavillano B, Di Mitri R, et al. Fully covered self-expandable metallic stents in benign biliary strictures: a multicenter study on efficacy and safety. Endoscopy 2012; 44: 923-927.
14. Tarantino I, Traina M, Mocciaro F, et al. Fully covered metallic stents in biliary stenosis after orthotopic liver transplantation. Endoscopy 2012; 44: 246-250.

15. Kahaleh M, Brijbassie A, Sethi A, et al. Multicenter trial evaluating the use of covered self-expanding metal stents in benign biliairy strictures: time to revisit our therapeutic options? J Clin Gastroenterol 2013; 47: 695-699.

16. Tuvignon N, Liguory $\mathrm{C}$, Ponchon $\mathrm{T}$, et al. Long-term follow-up after biliary stent placement for postcholecystectomy bile duct strictures: a multicenter study. Endoscopy 2011; 43: 208-216. 